\section{Asthma-Schulung auf die Spitze getrieben}

L uftiku(r)s ist ein Schulungsprogramm für asthmakranke Kinder und Jugendliche, das inzwischen bundesweit von den meisten Kassen erstattet wird. Im Mittelpunkt steht die intrafamiliäre Bewältigung der chronischen Krankheit. Dieses Konzept auf die Spitze getrieben hat Dr. Thomas Adelt, indem er vergangenen Sommer als Schulungsort die 2.032 Meter hoch gelegene Osnabrücker Hütte in Kärnten wählte. Die Vorteile liegen auf der Hand: quasi allergenfreie Umgebung, entspannte Urlaubsatmosphäre und trotzdem wenig Ablenkung, intensives Gruppenerlebnis. In Zusammenarbeit mit dem Deutschen Alpenverein konnte sogar die richtige Inhalations-

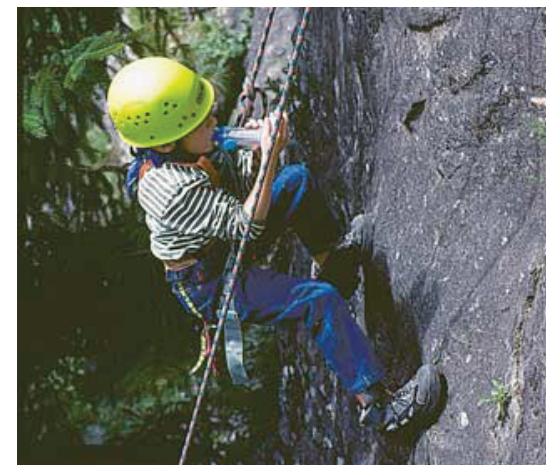

technik im Seil hängend geübt werden.

Kontakt: T. Adelt, Tel. o 5461 / 933 39,

E-Mail: stehling.adelt@t-online.de

\title{
Ekzem-Gefahr für Golden Girls
}

\begin{abstract}
old ist - zumindest in den USA für mehr Kontaktallergien verantwortlich als bisher vermutet. Die retrospektive Auswertung von 4.101 PatchTests bei Patienten mit Allergie-Verdacht aus den Jahren 1996 bis 1998 ergab 388 Sensibilisierungen gegen das Edelmetall. Dies entspricht innerhalb dieser vorselektierten Gruppe einer Prävalenz von 9,5\%. Damit hat Gold in der Hitliste der metallinduzierten TypIV-Allergien Kobalt (9,0\%) verdrängt und liegt hinter Nickel (14,2\%) auf Platz zwei. Manifestationsorte der Gold-Dermatitis waren vor allem Hand (Ringfinger), Gesicht und Augenlider. Betroffen waren fast ausschließlich Frauen (90,2\%), bei rund der Hälfte lag noch eine weitere Metall-Allergie vor. es
\end{abstract}

Fowler J et al. Am J Contact Dermat 2001; $12: 3-5$.

\section{Israelisch-Deutsche Gesellschaft gegründet} nuss-Allergie reagieren offenbar bei der ersten Exposition. Könnte Stillen hier die Allergie angebahnt haben? Ja - sagen Wissenschaftler aus Kanada. Bei elf von

n Nordamerika greift die ErdnussAllergie um sich: Erdnüsse sind dort inzwischen der häufigste Auslöser tödlicher Nahrungsmittel-induzierter Anaphylaxien. $80 \%$ der Kinder mit Erd-
23 Frauen war die Muttermilch nach dem Genuss von Erdnüssen Allergenhaltig. Danach sollten Mütter von Kindern mit erhöhtem Risiko Erdnüsse während der Stillzeit meiden, um eine Sensibilisierung des Säuglings zu verhindern.

Vadas $P$ et al. JAMA 2001; 285: 1746-8.

\section{Macht Margarine Männer zu Atopikern?}

\begin{abstract}
er Konsum mehrfach ungesättigter, pflanzlicher Fettsäuren fördert die IgE-Produktion und ist eine Ursache der in Industrieländern stetig steigenden Allergikerzahl - eine ebenso schöne wie gewagte These, die durch eine große epidemiologische Untersuchung aus Sachsen-Anhalt jetzt Unterstützung erfährt. Die Statistiker erfassten Daten von 2.128 Kindern im Alter zwischen fünf und 14 Jahren und unterteilten sie nach ihrem bevorzugten Brotaufstrich in Margarine- und ButterEsser. Während der Beobachtungszeit von einem Jahr protokollierten sie Symptome einer Rhinitis sowie Sensibilisierungen gegenüber gängigen Aeroaller-
\end{abstract}

genen mittels Bestimmung spezifischer IgE-Konzentrationen. In der Tat waren Sensibilisierungen bei Margarine bevorzugenden Jungen 1,57-fach häufiger als bei Butter-Essern, Symptome einer Rhinitis traten sogar 1,76-fach häufiger auf. Bei den Mädchen waren diese Zusammenhänge allerdings kaum ausgeprägt. Aufgrund der methodischen Beschränkungen sind die Ergebnisse der Studie natürlich mit Vorsicht zu genießen, der Konsum von Margarine scheint aber die bei Jungen ohnehin erhöhte Atopieneigung weiter zu fördern.

es

Bolte G et al. Am J Respir Crit Care Med 2001; 163: 277-9. sraelische und deutsche Professoren der Dermatologie haben eine gemeinsame Fachgesellschaft gegründet. Die erste Mitgliederversammlung fand während der 41. Tagung der Deutschen Dermatologischen Gesellschaft Anfang Mai statt. Die Gesellschaft möchte einerseits gemeinsame wissenschaftliche Projekte fördern, beispielsweise Gastaufenthalte junger Hautärzte und wissenschaftliche Symposien. Andererseits sieht sie ihre Aufgabe in der Aufarbeitung der Schicksale jüdischer Dermatologen und ihrer Bedeutung für die deutsche Dermatologie.

„Das große Interesse und die zahlreichen positiven Kommentare lassen auf eine rasche Entwicklung dieser Vorhaben hoffen und machen auch das Schaffen finanzieller Grundlagen wahrscheinlich“, sagte Prof. Dr. Wolfram Sterry in Berlin.

41. DDG-Tagung, Berlin 2001 